\title{
Serratia Marcescens Spinal Epidural Abscess Formation Following Acupuncture
}

\author{
Chih-Wei Yang ${ }^{1}$, Shun-Neng Hsu ${ }^{1}$, Jhih-Syuan Liu ${ }^{1}$ and Dueng-Yuan Hueng ${ }^{2,3}$
}

\begin{abstract}
The formation of spinal epidural abscess following acupuncture is very rare. We herein report the case of a 54-year-old woman who presented with progressive low back pain and fever with a root sign. She underwent surgical decompression, with an immediate improvement of the low back pain. A culture of the epidural abscess grew Serratia marcescens. One year postoperatively, magnetic resonance imaging revealed the almost complete eradication of the abscess. This case is the first case of Serratia marcescens-associated spinal epidural abscess formation secondary to acupuncture. The characteristics of spinal epidural abscess that develop after acupuncture and how to prevent such complications are also discussed.
\end{abstract}

Key words: spinal epidural abscess, acupuncture, Serratia marcescens

(Intern Med 53: 1665-1668, 2014)

(DOI: 10.2169/internalmedicine.53.1620)

\begin{abstract}
Introduction
Epidural abscess is an uncommon complication of acupuncture that may present with neurologic symptoms, including back and leg pain and numbness (1). Serratia marcescens is rarely reported to be a pathogen of invasive diseases in patients with acupuncture-treated low back pain. Moreover, Serratia marcescens is commonly opportunistic, affecting immunocompromised patients or those with severe medical comorbidities, such as diabetes mellitus (2). This study reports the rare case of a 54-year-old woman with no identifiable risk factors who presented with an epidural abscess at the lumbar region following acupuncture. The lesion was quickly diagnosed using T1-weighted gadoliniumenhanced magnetic resonance imaging (MRI). In acupuncture patients with neurologic symptoms, such as back pain, a root sign and fever, performing diagnostic MRI of the lumbar spine may help to identify peripsoas and spinal epidural abscess (1). To date, this is the first case of invasive Serratia marcescens-related spinal epidural abscess formation in a patient without previous medical comorbidities who underwent acupuncture (2).
\end{abstract}

Case Report

A 54-year-old woman consulted our department for an evaluation of progressive low back pain and fever lasting for two weeks. She had a hypertrophic intervertebral disc of the lumbar spine with numbness of the right lateral thigh and had been treated with acupuncture over the lumbar region twice a month for the past three months at a local hospital. One month prior to the consultation, she began to have low back pain. She had also experienced an intermittent fever (temperature: $37.7^{\circ} \mathrm{C}$ ) approximately two weeks earlier. Her low back pain had progressed and radiated to the right buttock and thigh, despite the acupuncture treatment.

On admission, the patient was alert and communicative. Her body temperature was $37.6^{\circ} \mathrm{C}$, her blood pressure was $135 / 88 \mathrm{mmHg}$ and her pulse rate was 76 beats per minute. A neurologic examination disclosed sensory deficits in the right lateral thigh. A complete blood count disclosed the following findings: total WBC count $=7,700 / \mu \mathrm{L}$ (normal, 4,000-11,000 $\mu \mathrm{L})$, hemoglobin $=13.1 \mathrm{~g} / \mathrm{dL}(13.5-17 \mathrm{~g} / \mathrm{dL})$ and platelet count $=313,000 / \mu \mathrm{L}(150,000-400,000 / \mu \mathrm{L})$. The laboratory data revealed an elevated C-reactive protein level

\footnotetext{
${ }^{1}$ Department of Internal Medicine, Tri-Service General Hospital, National Defense Medical Center, Taiwan, R.O.C., ${ }^{2}$ Department of Neurological Surgery, Tri-Service General Hospital, National Defense Medical Center, Taiwan, R.O.C. and ${ }^{3}$ Department of Biochemistry, National Defense Medical Center, Taiwan, R.O.C.

Received for publication August 22, 2013; Accepted for publication September 30, 2013

Correspondence to Dr. Dueng-Yuan Hueng, hondy2195@yahoo.com.tw
} 


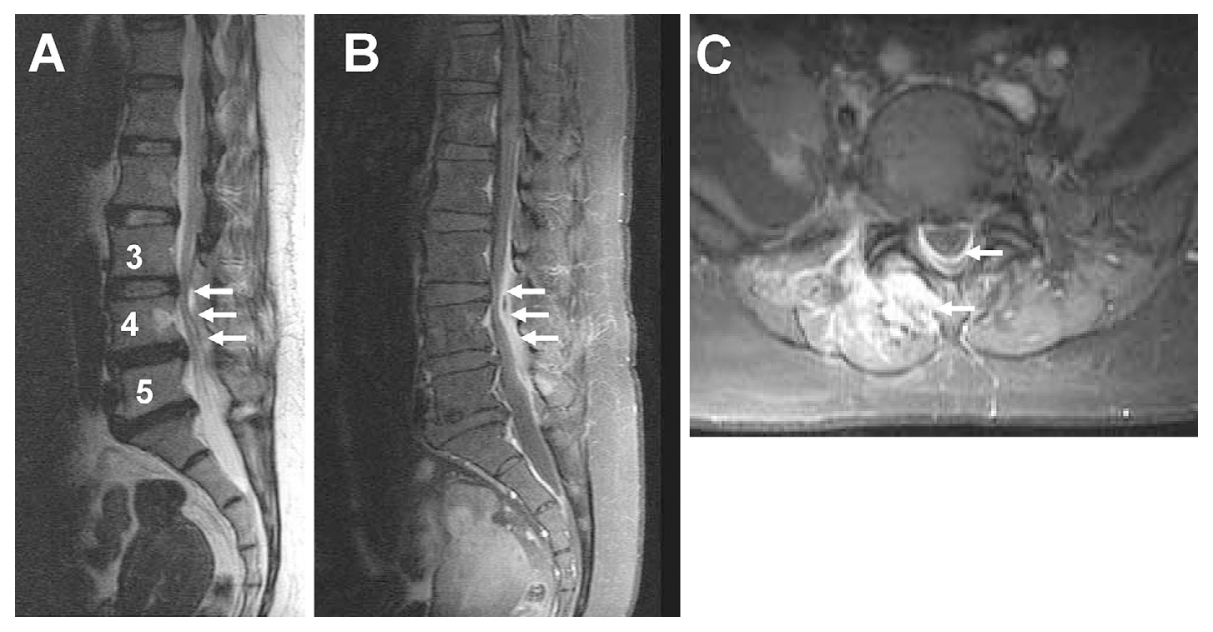

Figure 1. Preoperative magnetic resonance imaging (MRI). (A) T2-weighted MRI on the sagittal view showed extradural fluid collection at the level of L3-L5. (B) T1-weighted gadolinium-enhanced MRI demonstrated a contrast-enhanced lesion from L3 to L5 in the posterior epidural space. (C) Axial gadolinium-enhanced T1-weighted MRI revealed increased enhancement in the epidural space and right posterior paravertebral region.


Figure 2. Follow-up MRI performed one year postoperatively revealed the status post-laminectomy at L3-4. (A and B) There was no contrast-enhanced residual inflammatory fluid collection in the epidural space or the $(C)$ paraspinal soft tissue.

$(1.87 \mathrm{mg} / \mathrm{dL})$ and erythrocyte sedimentation rate (ESR) (72 $\mathrm{mm} / \mathrm{hr})$.

A T2-weighted MRI showed fluid collection at the L3 to L5 levels (Fig. 1A). T1-weighted gadolinium-enhanced MRI demonstrated contrast-enhanced fluid collection at the same level, consistent with the presence of a spinal epidural abscess causing severe spinal canal compression at the L3-4 level (Fig. 1B). Axial view MRI also revealed a contrastenhancing mass over the right posterior paravertebral region (Fig. 1C).

The patient underwent left total laminectomy of L3-4 to decompress the spinal epidural abscess, which was diagnosed based on the yellowish abscess found intraoperatively in the epidural space from L3 to L4. The patient was initially treated with oxacillin. Two days after the surgery, the culture of the abscess yielded Serratia marcescens. The iso- lated Serratia marcescens was sensitive to piperacillin/tazobactam, ceftriaxone, cefepime and ertapenem (minimum inhibitory concentration (MIC) $\leq 16 / 4 \mu \mathrm{g} / \mathrm{mL}$, MIC $\leq 1 \mu \mathrm{g} / \mathrm{mL}$, MIC $\leq 8 \mu \mathrm{g} / \mathrm{mL}$ and MIC $\leq 0.5 \mu \mathrm{g} / \mathrm{mL}$, respectively). The patient received intravenous ceftriaxone ( $2 \mathrm{~g}$ every 12 hours for eight weeks) to treat the infection and began recovering three days after the start of the antibiotic treatment. The symptoms of low back pain and numbness over the right thigh improved after eight weeks of treatment. Three months after completing the antibiotic therapy, the patient's ESR returned to normal. She recovered well without any neurologic sequelae. Follow-up MRI performed one year later revealed no residual or recurrent inflammatory fluid collection in the epidural space or paraspinal soft tissue (Fig. 2). 
Table. Articles Related to Spinal Epidural Abscess Formation Following Acupuncture Published between 1998 and 2013

\begin{tabular}{cccccc}
\hline Reference & $\begin{array}{c}\text { Age (yrs), } \\
\text { Gender }\end{array}$ & Spine Level & Clinical manifestations & Pathogen & Treatment \\
\hline 5 & $67, \mathrm{M}$ & C1-2 & low-grade fever and backache & no specific pathogen \\
14 & 64, M & T11-L3 & severe back pain & Escherichia coli & antibiotics \\
15 & 13, M & L4-5 & acute severe back pain and fever & no specific pathogen \\
16 & $47, \mathrm{M}$ & C1-3 & posterior nuchal pain, and swelling & no specific pathogen \\
17 & 19, M & C2-6 & progressive neck stiffness and fever & Group B Streptococcus & antibiotics \\
Current case & $47, \mathrm{M}$ & L3-5 & fever, low back pain, and root sign & Serratia marcescens & decompression + antibiotics \\
\hline
\end{tabular}

\section{Discussion}

This study reports a rare case of spinal epidural abscess formation following acupuncture, a common procedure in traditional Chinese medical practice. In general, acupuncture is one of the early forms of medical intervention that originated from China thousands of years ago. Some reviews and studies have disclosed that acupuncture has clinical value for chronic pain, nausea, vomiting, headaches and hypertension. Major complications are uncommon (3), with an incidence of approximately 0.55 per 10,000 individual patients in 12 prospective surveys (4). One cumulative review of 715 major complications of acupuncture revealed that trauma is common $(276,38 \%)$, as well as infection $(295,41 \%)$. The most common infection is hepatitis B $(>60 \%)$ due to transmission via needle puncture. However, there are only three cases of spinal infection (4).

One case report stated that cervical spinal epidural abscess formation is associated with acupuncture (5). Completely sterile conditions are difficult to maintain in the clinical practice of acupuncture. The efficacy of skin disinfection before acupuncture needle insertion is debatable, and the unintentional inoculation of skin surface microorganisms is possible (6). In healthy individuals, resident microorganisms present on the skin surface cannot easily cause clinical infections. However, bacteria that are not part of the normal skin flora have a higher potential of causing infection (6).

Spinal epidural abscess is also uncommon, with an incidence of approximately one per 20,000 hospital admissions (7), although the frequency has increased in the last two decades due to the improved sensitivity of MRI and the increased use of aggressive spinal procedures and operative devices (7). Predisposing risk factors include diabetes mellitus, chronic kidney disease, alcoholism, an immunocompromised status, trauma, surgery, tattooing, nerve acupuncture and infection (i.e., sepsis, soft tissue infection, osteomyelitis and urinary tract infection) (7). The most common causative pathogen is Staphylococcus aureus, which accounts for approximately two-thirds of cases (7). In a meta- analysis of patients with spinal epidural abscess, Gramnegative bacteria had been found to have Escherichia coli (8) and Pseudomonas aeruginosa (9). Culture-confirmed Serratia marcescens as the cause of a spinal epidural abscess is very uncommon, although some reports have disclosed such cases (10-13). One case involved communityacquired infection (2), while another was associated with secondary disseminated infection (12). Most cases of Serratia marcescens spinal epidural abscess are related to postneurosurgical intervention. On the other hand, only five cases of spinal epidural abscess formation following acupuncture have been published since 1998 (14-17). The features of all five reported cases are listed in Table. Importantly, the characteristics of spinal epidural abscess that develop following acupuncture include puncture site-specific features and procedure-related pathogens, in contrast to other types of spinal epidural abscess. Possible explanations for why this patient developed a Serratia marcescens spinal epidural abscess after undergoing acupuncture include the invasiveness of the acupuncture procedure and the possibility of Serratia marcescens colonization and infection from a healthcare worker due to the long-term presence of the bacteria on the hands (18) and the inadequate use of nonmedicated liquid soap (19).

Serratia marcescens belonging to the Enterobacteriaceae family is a Gram-negative bacillus that has been recognized to be a pathogen of nosocomial infections over the past 20 years (20). Hospital-acquired Serratia marcescens infection has various causes, including contaminated medical devices, contaminated fluid and cleaning solution and the contaminated hands of hospital staff (21). The infections usually involve the urinary tract, respiratory tract or wounds and may lead to septicemia, although infection of the central nervous system (CNS) has rarely been reported (20). The major risk factor for Serratia marcescens CNS infection is a postneurosurgical state because breaking the patient's CNS defenses provides the opportunity for the transmission of an infection from the hospital environment. A high mortality rate of greater than $50 \%$ has been reported due to inadequate recognition and treatment (10). 
In the present case, the patient had a history of undergoing needle acupuncture several times and lacked other risk factors associated with spinal epidural abscess. In the present case, local acupoints GV 3 (Yaoyangguan) of the posterior lumbar region were selected for relief of persistent low back pain. Yaoyangguan is located below the spinous process of the fourth lumbar vertebra (22), compatible with the location of the lesion found on lumbar MRI and during surgery. The pus and cerebrospinal fluid (CSF) cultures grew Serratia marcescens, a rare pathogen of spinal epidural abscess. The patient underwent surgical decompression and completed antibiotic therapy. This case is striking because the development of Serratia marcescens spinal epidural abscess following acupuncture is very rare and has not been previously reported. In addition to vancomycin to cover methicillin-resistant Staphylococcus aureus (MRSA), a thirdor fourth-generation cephalosporin should be administered as soon as possible due to the high mortality rate of patients with Serratia marcescens CNS infection.

In patients who undergo acupuncture, hand washing by the acupuncturist and the use of the clean needle technique and disposable needles should be routinely practiced in order to minimize the risk of complications (23). The necessity of sterilizing the skin before acupuncture is controversial and debatable for healthy individuals; however, it should be performed in immunocompromised patients and the elderly (6). Although spinal epidural abscess is an uncommon disease, they should be included in the differential diagnosis in patients with this clinical presentation, especially in those treated with needle acupuncture, due to the high mortality of the condition. Aggressive treatment with surgical drainage and systemic empirical antibiotics must be initiated when a spinal epidural abscess is initially diagnosed. Serratia marcescens infection should be considered in the differential diagnosis if the symptoms and fever persist after the initial management. Once the initial antibiotic treatment fails, the patient should proceed to surgical drainage to obtain a culture report. A third-generation cephalosporin should then be used to treat spinal epidural abscess that develop following acupuncture when the pathogen Serratia marcescens and susceptibility results are identified.

The authors state that they have no Conflict of Interest (COI).

\section{References}

1. Heidemann J, Spinelli KS, Otterson MF, et al. Case report: magnetic resonance imaging in the diagnosis of epidural abscess complicating perirectal fistulizing Crohn's disease. Inflamm Bowel Dis 9: 122-124, 2003.

2. Parkins MD, Gregson DB. Community-acquired Serratia marcescens spinal epidural abscess in a patient without risk factors: Case report and review. Can J Infect Dis Med Microbiol 19: 250-252,
2008.

3. MacPherson $\mathrm{H}$, Thomas $\mathrm{K}$, Walters $\mathrm{S}$, et al. The York acupuncture safety study: prospective survey of 34000 treatments by traditional acupuncturists. BMJ 323: 486-487, 2001.

4. White A. A cumulative review of the range and incidence of significant adverse events associated with acupuncture. Acupunct Med 22: 122-133, 2004.

5. Yazawa $S$, Ohi $T$, Sugimoto $S$, et al. Cervical spinal epidural abscess following acupuncture: successful treatment with antibiotics. Intern Med 37: 161-165, 1998.

6. Hoffman P. Skin disinfection and acupuncture. Acupunct Med 19: 112-116, 2001.

7. Darouiche RO. Spinal epidural abscess. N Engl J Med 355: 20122020, 2006.

8. Chao D, Nanda A. Spinal epidural abscess: a diagnostic challenge. Am Fam Physician 65: 1341-1346, 2002.

9. Reihsaus E, Waldbaur H, Seeling W. Spinal epidural abscess: a meta-analysis of 915 patients. Neurosurg Rev 23: 175-204; discussion 205, 2000.

10. Huang $\mathrm{CR}, \mathrm{Lu} \mathrm{CH}$, Chien $\mathrm{CC}$, et al. Protean infectious types and frequent association with neurosurgical procedures in adult Serratia marcescens CNS infections: report of two cases and review of the literature. Clin Neurol Neurosurg 103: 171-174, 2001.

11. Kulkarni AG, Hee HT. Adjacent level discitis after anterior cervical discectomy and fusion (ACDF): a case report. Eur Spine J 15 (Suppl 5): 559-563, 2006.

12. Nicasio AM, Quintiliani R Jr, DeRyke CA, et al. Treatment of Serratia marcescens meningitis with prolonged infusion of meropenem. Ann Pharmacother 41: 1077-1081, 2007.

13. Perez-Toro MR, Burton AW, Hamid B, et al. Two-tuohy needle and catheter technique for fluoroscopically guided percutaneous drainage of spinal epidural abscess: a case report. Pain Med 10: 501-505, 2009.

14. Bang MS, Lim SH. Paraplegia caused by spinal infection after acupuncture. Spinal Cord 44: 258-259, 2006.

15. Ishibe M, Inoue M, Saitou K. Septic arthritis of a lumbar facet joint due to pyonex. Arch Orthop Trauma Surg 121: 90-92, 2001.

16. Lee JH, Cho JH, Jo DJ. Cervical epidural abscess after cupping and acupuncture. Complement Ther Med 20: 228-231, 2012.

17. Lee SY, Chee SP. Group B Streptococcus endogenous endophthalmitis: case reports and review of the literature. Ophthalmology 109: 1879-1886, 2002.

18. de Vries JJ, Baas WH, van der Ploeg K, et al. Outbreak of Serratia marcescens colonization and infection traced to a healthcare worker with long-term carriage on the hands. Infect Control Hosp Epidemiol 27: 1153-1158, 2006.

19. Sartor C, Jacomo V, Duvivier C, et al. Nosocomial Serratia marcescens infections associated with extrinsic contamination of a liquid nonmedicated soap. Infect Control Hosp Epidemiol 21: 196-199, 2000.

20. Hejazi A, Falkiner FR. Serratia marcescens. J Med Microbiol 46: 903-912, 1997.

21. Ostrowsky BE, Whitener C, Bredenberg HK, et al. Serratia marcescens bacteremia traced to an infused narcotic. N Engl $\mathrm{J}$ Med 346: 1529-1537, 2002.

22. Hu ML, Zhang RP. Clinical observation on warming-promotion acupuncture for lumbar muscle strain. Zhongguo Zhen Jiu 31: 622-624, 2011 (in Chinese, Abstract in English).

23. Lao L, Hamilton GR, Fu J, et al. Is acupuncture safe? A systematic review of case reports. Altern Ther Health Med 9: 72-83, 2003.

(C) 2014 The Japanese Society of Internal Medicine http://www.naika.or.jp/imonline/index.html 